\title{
Editorial
}

\section{Producción científica}

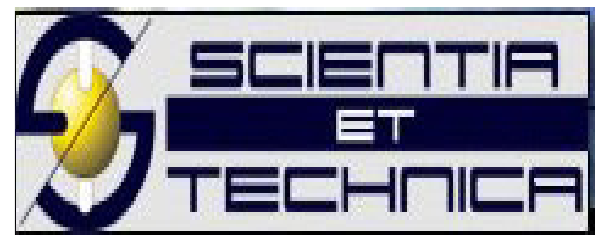

La producción científica expresada a través del número de publicaciones, es uno de los indicadores de mayor importancia a la hora de evaluar la actividad en un país, institución o investigador. Su evaluación mediante métodos y procedimientos matemáticos abordados desde la perspectiva de los estudios métricos de la información, arrojan resultados necesarios en la gestión de la investigación y en los procesos de toma de decisiones para el desarrollo de politicas científicas.

Por ello, la evaluación del impacto de la ciencia y la tecnología constituye una necesidad estratégica para constatar el desarrollo de un país, de su política científica y de su gestión en función de la sociedad.

Por la propia misión de las universidades es imprescindible que su producción científica sea objeto de reflexión y evaluación debido a los imperativos del desarrollo social [1]. El proceso científico se puede considerar similar a los modelos económicos coste-beneficio o inversiónresultado, por tanto, debe ser cuantificado.

En principio las inversiones en ciencia se pueden medir fácilmente al ser tangibles, analizando los recursos materiales $\mathrm{y}$ humanos con que se cuenta. Pero la evaluación de los resultados científicos no se ha resuelto todavía de forma definitiva, ya que supone el complejo mecanismo de 
medir el conocimiento generado en las tareas de investigación. A partir de esto ha surgido la necesidad de evaluar el rendimiento de la actividad científica y su impacto en la sociedad, con el fin de adecuar convenientemente la asignación de los recursos destinados a investigación y desarrollo [2].

A partir de un intercambio académico, entre el Centro de Referencia para la Educación de Avanzada-CREA, del Instituto Superior Politécnico "José Antonio Echeverría" y la Universidad Tecnológica de Pereira de Colombia, surgieron otras colaboraciones en el ámbito científico a través de sus publicaciones seriadas: la Revista Referencia Pedagógica-RRP (del CREA) y la Revista Scientia et Technica de la UTP).

Lo anterior favorece un incremento de las colaboraciones entre investigadores a través de la publicación de artículos científicos en temáticas afines a los dos centros universitarios.

Para ello es necesario realizar evaluaciones generales en cada una de sus publicaciones sobre el comportamiento de la producción científica, que permitan tomar decisiones para optimizar y reorientar sus investigaciones; racionalizar la organización de la investigación; reestructurar las investigaciones en determinados campos y aumentar la productividad de la investigación.

A partir de estas necesidades, el objetivo de la investigación es ofrecer una evaluación sobre el estado actual de la producción científica de las Revistas y proponer diversas acciones que contribuyan a posicionar la publicación en índices de mayores niveles de visibilidad.

\section{Hugo Armando Gallego Becerra Profesor Titular}

Universidad Tecnológica de Pereira

Editor en Jefe

Revista Scientia Et Technica

\section{Juán Francisco Cabrera Ramos}

Comité Editorial - Scientia Et Technica

Centro de Referencia para la Educación de Avanzada (CREA).

La Habana Cuba 


\section{REFERENCIAS}

[1]. Ortiz Torres, E., et al., Evaluación del impacto científico de las tesis doctorales en Ciencias Pedagógicas mediante indicadores cienciométricos. Revista Española de Documentación Científica, 2010. 33(2): p. $279-286$.

[2]. González de Dios, J., M. Moya, and M.A. Mateos Hernández, Indicadores bibliométricos: Características y limitaciones en el análisis de la actividad científica. Anales Españoles de Pedriatría, 1997. 47: p. 235 - 244.

[3]. Ortiz Torres, E., et al., Evaluación del impacto científico de las tesis doctorales en Ciencias Pedagógicas mediante indicadores cienciométricos. Revista Española de Documentación Científica, 2010. 33(2): p. 279-286.

[4]. González de Dios, J., M. Moya, and M.A. Mateos Hernández, Indicadores bibliométricos: Características y limitaciones en el análisis de la actividad científica. Anales Españoles de Pedriatría, 1997. 47: p. 235-244.

[5]. Hernández Sampieri, R., Metodología de la Investigación. 2008, La Habana: Félix Varela. 475. 